\title{
52 CLOSTRIDIUM DIFFICILE ENTERITIS COMPLICATING UNDIAGNOSED COELIAC DISEASE, A NEW CLINICAL PROBLEM?
}

J A Gray, F Mullan, P B Allen Departments of Gastroenterology and General Surgery, Causeway Hospital, 4 Newbridge Road, Coleraine, Northern Ireland

\subsection{6/gutjnl-2013-305143.52}

Introduction Clostridium difficile (CD) represents the leading cause of health care associated infective diarrhea in the UK. Normally manifesting as infectious colitis, small bowel involvement known as $\mathrm{CD}$ enteritis is a very rare condition.

We present the first documented case in the literature of $\mathrm{CD}$ enteritis complicating previously undiagnosed coeliac disease.

A 63 year old male with no significant past medical history presented with a 3 month history of abdominal pain, bloating, weight loss, intermittent diarrhoea and night sweats. He was prescribed no regular medications, however had a course of antibiotics 6 weeks previous for a confirmed UTI. Blood results

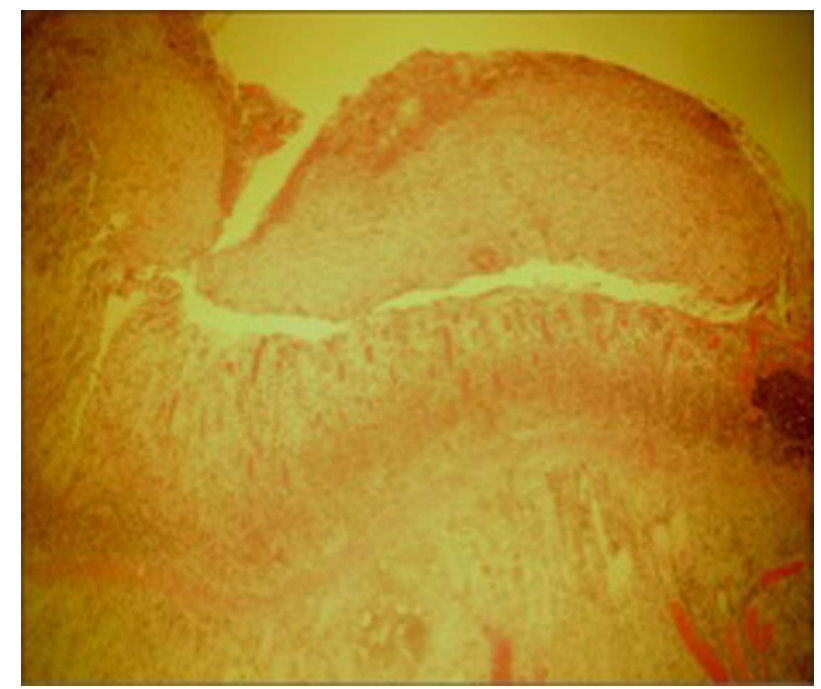

Figure 1 Small bowel resection showing ulceration/pseudomembrane and gran positive bacillus.

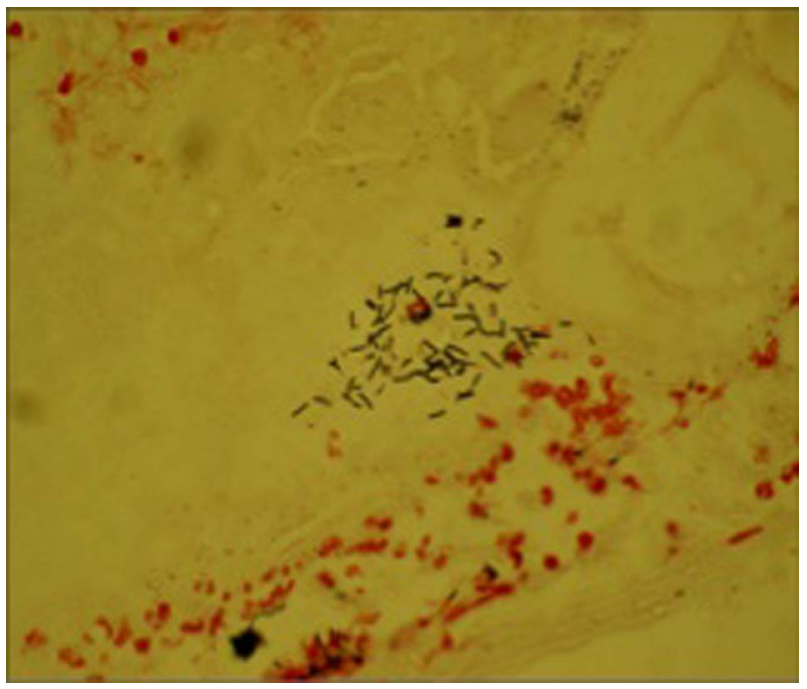

Figure 2 Gram positive bacillus. 


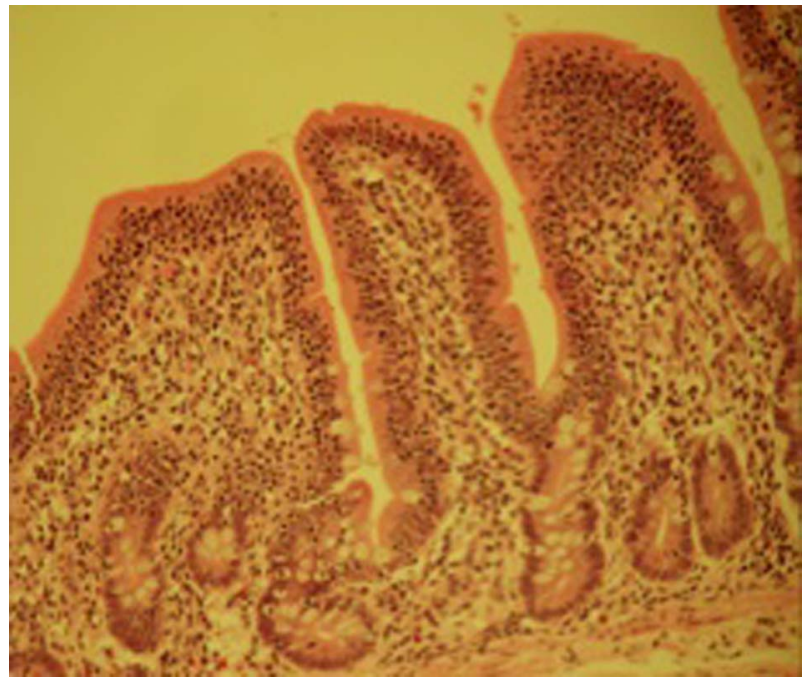

Figure 3 Proximal area of small bowel resection showing partial vitlous atrophy and increased intra-epithelial lymphocytosis.

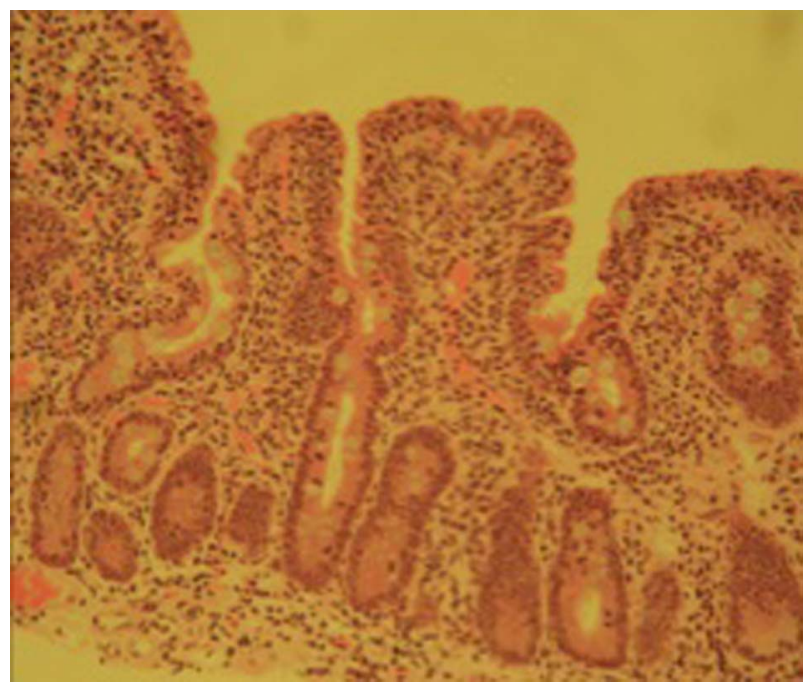

Figure 4 Subsequent duodent biopsy at time of follow up OGD confirming villous atrophy and intra-epithelial lymphocytosis.

revealed WCC 49 (4-11), PLTs 597 (150-400), CRP 86 (0-5) and albumin $22(35-50)$.

A CT scan of abdomen showed a short segment of small bowel thickening with adjacent lymphadenopathy. The differential diagnosis included Crohns disease, small bowel lymphoma and carcinoid tumour.

Due to worsening pain he underwent diagnostic laparotomy, with small bowel resection revealing severe mucosal ulceration and pseudomembranous lining. Histology identified grampositive rods in the areas of marked ulceration with proximal subtotal villous atrophy and marked intra-epithelial lymphocytosis. Stool samples confirmed presence of CD toxin.

Coeliac serology revealed anti-TTG levels of 29.4 (0-15). Duodenal biopsies confirmed coeliac disease. The patient improved with a 14 day course of oral metronidazole and commencement of a gluten free diet.
Conclusion This case highlights the importance of considering extra-colonic manifestations of CD especially in those with risk factors including increasing age, comorbidities, IBD and recent antibiotic use. 